\title{
Use of an electronic medication management support system in patients with polypharmacy in general practice: study protocol of a quantitative process evaluation of the AdAM trial
}

Robin Brünn ( $\nabla$ bruenn@allgemeinmedizin.uni-frankfurt.de )

Goethe-Universitat Frankfurt am Main

Dorothea Lemke

Goethe-Universitat Frankfurt am Main

Kiran Chapidi

Goethe-Universitat Frankfurt am Main

Juliane Köberlein-Neu

University of Wuppertal: Bergische Universitat Wuppertal

\section{Alexandra Piotrowski}

University of Wuppertal: Bergische Universitat Wuppertal

\section{Sara Söling}

University of Wuppertal: Bergische Universitat Wuppertal

\section{Wolfgang Greiner}

Bielefeld University: Universitat Bielefeld

\section{Petra Kellermann-Mühlhoff}

BARMER GEK

\section{Nina Timmesfeld}

Ruhr-Universität Bochum: Ruhr-Universitat Bochum

\section{Marjan van den Akker}

Goethe-Universitat Frankfurt am Main

Christiane Muth

Goethe-Universitat Frankfurt am Main

\section{Study protocol}

Keywords: Polypharmacy, study protocol, process evaluation, decision support, primary care

Posted Date: April 12th, 2021

DOI: https://doi.org/10.21203/rs.3.rs-392792/v1 
License: (c) (i) This work is licensed under a Creative Commons Attribution 4.0 International License. Read Full License

Version of Record: A version of this preprint was published at Therapeutic Advances in Drug Safety on January 1 st, 2022. See the published version at https://doi.org/10.1177/20420986211073215. 


\section{Abstract}

Background: Much research is currently devoted to polypharmacy, but interventional studies often fail to significantly improve patient-relevant outcomes or just measure surrogate parameters. Interventions and their settings are often complex, and a wide range of factors affect results. The aim of the AdAM study is to reduce hospitalization and death by requiring general practitioners (GPs) to use a computerized decision-support system (CDSS). The study will undergo a process evaluation to identify relevant factors for successful implementation and to assess whether the intervention was implemented as intended.

Research questions: We will assess implementation (reach, fidelity, dose, tailoring) by asking the following questions: (1) Who took part in the intervention, i.e., the proportion of GPs that used the CDSS, and the proportion of patients that were enrolled by them? Information on the characteristics of GPs and patients will also be collected. (2) How many and what type of medication alerts were handled by the GPs? (3) Was the intervention implemented as intended? (4) On what days did GPs use the intervention tool?

We will conduct subgroup analyses to all questions, including time effects to account for software improvements over time.

Methods: The process evaluation is part of a stepped wedge cluster-randomized controlled trial. Characteristics of practices, GPs and patients using the CDSS will be compared with the non-participating population. CDSS log data will be analyzed to evaluate how the number of medication alerts changed between baseline and two months later and identify the kind of medication alerts that are given highest priority. The comparison of enrolled patients on weekdays vs weekends will shed light on whether GPs use the CDSS in the absence or presence of patients. All outcomes will be presented using descriptive statistics, and significance tests will be used to identify any associations between them.

Discussion: This study protocol is the basis for conducting the analyses of the log data-based process evaluation. By providing insight into the way GPs conduct medication reviews, the evaluation will provide a context to the trial results and support their interpretation. The evaluation relies on the proper documentation of what the CDSS was used for, which may limit its explanatory power.

Trial registration: ClinicalTrials.gov, NCT03430336. Registered on February 6, 2018. https://clinicaltrials.gov/ct2/show/NCT03430336

\section{Contributions To The Literature}

- The intervention is the first one to use log data of a computerized decision-support system.

- This study will help explain the processes general practitioners go through when performing a medication review. It will shed light on circumstances that facilitate or hinder the success of the implementation of a computerized decision-support system. 
- A preplanned study protocol defines a priori the analyses that will be conducted to fulfill reporting standards.

\section{Background}

As a result of improvement in medical treatments for formerly fatal chronic diseases, life expectancy, and the number of people with multiple chronic conditions (multimorbidity), has increased throughout the world.[1] Multimorbidity is associated with polypharmacy, the concurrent use of multiple drugs by a patient. There is no consensus on the definition of polypharmacy, but it is commonly defined as the daily intake of at least five different drugs.[2] Polypharmacy has become more prevalent over the years, with estimates of the number of affected patients ranging from one in five adults [3] to more than half the older population (65+).[4] As large numbers of drugs may be required to treat multiple diseases, polypharmacy may often be appropriate. Nonetheless, inappropriate therapy regimens, with unrecognized duplicate prescriptions or drug-drug and drug-disease interactions may lead to therapy failure, deterioration in conditions or effect reinforcement.[5]

In order to manage patients' medication and avoid inappropriate polypharmacy, many clinical trials, with or without computerized support, have been conducted to examine the effectiveness of medication reviews in recent years. The findings of these trials have been inconsistent and have often failed to generate significant results. In particular, beneficial effects of medication reviews on patient-relevant outcomes could not be proven.[6, 7]

Furthermore, as practices are complex systems in themselves, it is not only the interventions that are often complex. [8] This implies that many factors influence the measurable outcomes. By assessing factors that may influence intervention outcomes and determining whether the intervention is implemented in the target population as intended, process evaluations can show how the intervention works. This can facilitate the interpretation of results and provide insight into reasons for success or failure. The underlying theoretical framework based on a proposal by Wierenga et al [9] can be found in

Fig. 1. The most important domains of a process evaluation are Reach (who received the intervention and who did not?), Dose (how intense was the intervention?), Fidelity (was the intervention delivered as intended?), and Tailoring (how was the intervention altered to imbed it in a daily routine?).[10]

In the AdAM study ("Anwendung für ein digital gestütztes Arzneimitteltherapie- und Versorgungsmanagement", or "application of digitally supported drug-therapy and care management"), the electronic medication management system "eMMa" is used by general practitioners (GPs). In this paper, we describe the process evaluation of the AdAM study, based on log data from eMMa.

\section{The AdAM study}

The primary aim of the AdAM study is to determine whether a yearly medication review supported by eMMa effectively reduces the combined endpoint of all-cause hospital admissions and all-cause mortality in adult patients with five or more chronic prescriptions in primary care. The evaluation of 
eMMa assesses cost-effectiveness, physicians' and patients' perspectives on the intervention, a sustainability assessment, a qualitative comparative analysis of contextual and implementation process factors, and the process evaluation described in this paper.

Design, setting and population: The full details of the trial design and its methodology will be published elsewhere. Briefly, the AdAM study is a stepped-wedge cluster-randomized controlled trial (CRCT) with open cohorts conducted in general practices in the German region of Westphalia-Lippe from 2017 to 2021. General practices are the units of randomization and all practice patients are treated in accordance with the practice's group assignment. General practices are invited to participate when one or more physicians in the practice is a GP, an internist, or a physician without specialization that provides primary care in accordance with the KVWL (Kassenärztliche Vereinigung Westfalen-Lippe, a regional Association of Statutory Health Insurance Physicians) and that claims data shows to have at least eleven potentially eligible patients. Every month, newly recruited general practices are randomized into either the intervention group or the waiting control group using block randomization of variable block length based on practice IDs. In total, the target number of included practices was 1080. The statutory health insurance company BARMER provides randomized practices in the intervention group with lists of potentially eligible patients based on claims data. As the lists are updated quarterly, the cohort is open. Eligible patients are 18 years or older, have five or more chronic prescriptions (at least five different codes according to the Anatomical Therapeutic Chemical Classification System in two subsequent quarters) and are insured by BARMER. They are invited to participate by general practices in the intervention group and are enrolled in AdAM after giving their written informed consent. They are then provided with the experimental treatment foreseen for the intervention group. BARMER also generates lists of potentially eligible patients at general practices in the waiting control group based on claims data, but do not disclose this information to the practices, which continue to provide usual care. After 15 months in the waiting control group, these practices also receive a list of potentially eligible patients and updated quarterly lists of potentially eligible patients are disclosed to them from then on. Patients on the lists are invited to participate and enrolled in AdAM after giving written informed consent. They then also receive the experimental intervention.

Experimental intervention: Supported by eMMa, GPs conduct medication reviews for patients in the intervention group at least once a year. eMMa is unlocked for general practices in the intervention group, while general practices in the waiting control group have no access during the 15-month control period. Practice patients that are potentially eligible for AdAM are identified in eMMa. After a patient is enrolled, the practice receives access to their entire claims data, including diagnoses, prescriptions, and other data on health services utilization such as hospital stays and contacts with other physicians. eMMa provides the opportunity to update information (e.g., on new diagnoses and prescriptions for which claims have not yet been made) and to add specific details that are not included in claims data (e.g., height, weight, laboratory test results on kidney function, over-the-counter drugs, and medication doses). GPs then examine patients' medication regimens, supported by alerts from eMMa in case of inappropriate prescriptions (e.g., drug-drug and drug-disease interactions, inappropriate dosages, or potentially inappropriate medication because of age). 
Alerts are provided by eMMa according to a four-level system denoting severity ("red" for severe, "yellow" for less critical medication alerts, "grey" for alerts that are probably not clinically relevant, "info" for informative content). The severity rating in eMMa is based on continuous screening of medical publications and the notifications of German and international regulatory authorities provided by physicians and pharmacists. The sources are systematically analyzed in accordance with the structured WHO UMC algorithm for the categorization of the causality of adverse drug reactions.[11] Quality of evidence is based on the GRADE system.[12]

GPs can optimize treatment accordingly or document reasons for not changing a potentially inadequate medication (PIM), save the changes in eMMa, print a medication plan, and discuss changes with the patient. This optimization process can be carried out with or without the patient present. The trial compares outcomes at baseline and after two months. Once per year, physicians receive an annual reimbursement of $€ 85$ for each patient treated using eMMa.

Intervention training and support: General practice teams are invited to a two-hour continuous medical education (CME) session on polypharmacy and the main functions of eMMa on a voluntary basis. CME was provided by consulting pharmacists and IT specialists familiar with eMMa at two central locations (Münster and Dortmund), as well as in decentralized KVWL district offices. In addition, FAQ and training videos are provided on the KVWL website. Support hotlines for questions relating to administration, IT and polypharmacy are accessible for GPs upon request. KVWL contacts participating general practices in case of low numbers of visits to the eMMa site, or low rates of enrolled patients, via fax or by phone.

Outcomes: Apart from the combined primary outcome hospitalization or death from any cause, a number of secondary outcomes will be assessed: (i) indicators of high-risk prescribing and (ii) specific-cause hospital admissions preceded by high-risk prescribing (e.g. increased risk of gastrointestinal bleeding due to prescription of oral anticoagulants with either non-steroidal anti-inflammatory drugs (NSAIDs) or platelet aggregation inhibitors without a gastroprotective drug, increased cardiovascular risk due to prescription of oral NSAIDs for either heart failure patients or those receiving angiotensin-converting enzyme inhibitors $[13,14]$ ), as well as (iii) process measures, such as number of potentially inappropriate medications and underuse of medication (START criteria[15]).

\section{Methods}

\section{Aims and Objectives of the process evaluation of the AdAM study}

The process evaluation of the AdAM study accompanies an evaluation of the effectiveness of the experimental intervention eMMa, a computerized decision-support system (CDSS). In accordance with the UK's Medical Research Council guidelines[10], our process evaluation will determine whether the intervention was implemented as intended by assessing the following dimensions and questions:

- Reach: The question behind this dimension is: Was the recruitment of patients, GPs, and general practices successful? For this purpose, the study population that was enrolled in eMMa is compared 
with the eligible but non-enrolled population. Figure 1 provides an overview of the definition of the populations under consideration.

- Dose: The overarching question in this dimension deals with how comprehensively the intervention is applied. For this purpose, we examine the number, as well as the type (prioritization), of alerts that were responded to, and those that were not.

- Fidelity: Was the intervention implemented in such a way that success was possible? To achieve this, we defined several parameters assessed for the "Dose" domain that we regarded as crucial to the intervention's success and mandatory if the intervention was to be considered implemented as intended.

- Tailoring: How did physicians ensure the intervention fitted in well with their daily routine? We will assess the temporal dimensions (each day of the week as well as a comparison between working days and weekends) of eMMa usage.

\section{Data and methods of the process evaluation}

\section{Time}

Like the trial evaluation, data is collected at two time points, at baseline $\left(T_{0}\right)$, and two months later. $T_{0}$ is defined as the moment when the anamnesis has been completed and confirmed by the GP, after which he or she will be able to see the alerts provided by eMMa. If this is not confirmed, surrogate triggers will function as $T_{0}$, depending on any information entered into eMMa (e.g., adding missing dosage, input of physical parameters). Sensitivity analyses will be conducted for patients with a surrogate $T_{0}$.

\section{Population}

Different study populations are defined for the four dimensions as follows:

- For the Reach dimension, the enrolled study population consisting of patients, GPs, and general practices (= intervention group) are compared with their non-participating counterparts at $\mathrm{T}_{0}$.

Patients: The population of enrolled patients is compared with the eligible (non-enrolled) BARMER population, as well as with those patients that gave their informed consent for AdAM but have no activity documented in the eMMa software (= inactive population).

GPs: The enrolled GPs are compared with the eligible GP population in the study region, as well as with those GPs that gave their informed consent for AdAM but have no patient with documented activity in the eMMa software (= inactive population).

General practices: The enrolled general practices are compared with the eligible general practice population in the study region, as well as with those practices that gave their informed consent for AdAM but without any patient with documented activity in the eMMa software (= inactive population). 
- Dose \& Fidelity: The enrolled patient population (= intervention group) is defined as the study population and comparisons are made between $T_{0}$ and $T_{1}$.

- Tailoring: The enrolled GP population (= intervention group) is defined as the study population at $\mathrm{T}_{0}$.

\section{Outcomes.}

Reach: The main focus here is on group comparisons (e.g., proportions, mean values):

- Patients (Is there a prioritization in patient recruitment?):

Proportion of the enrolled patient population vs eligible (non-enrolled) BARMER population vs inactive patient population.

Group differences (enrolled vs non-enrolled vs inactive patient population) in terms of patient characteristics (e.g., age, gender, disease score, economic status).

We will evaluate whether there is an association between the enrollment rate of patients and disrupting factors. These factors will be the seasonal influenza wave, the COVID-19 pandemic, and technical difficulties that made it temporarily impossible to access the software. A time protocol including software updates and technical problems will be provided by KVWL and BARMER. GPs (Was there a selection bias in GP recruitment?):

Proportion of GPs using eMMa compared to the overall GP population in the study region and the inactive GP population.

Group differences (enrolled vs overall vs inactive) in terms of GP characteristics (e.g., age, sex, specialization).

General practice (Did selection bias exist in the recruitment at the general practice level?):

Proportion of general practices using eMMa compared to the entirety of practices in the study region and the inactive GP population.

Group differences (enrolled vs overall vs inactive) in terms of practice characteristics (e.g., employed GPs, number of patient visits)

A detailed overview of the outcome variables is provided in the data collection section.

Dose: To assess the intervention dose, the summed differences in alerts per GP (representing the number of patients treated in eMMa) at $\mathrm{T}_{0}$ compared to $\mathrm{T}_{1}$ will be calculated. We will also assess how the number of alerts per patient changes from $T_{0}$ to $T_{1}$. Seven different alert categories (see basic alerts in Fig. 1) are registered by eMMa, with up to four severity levels for each. This process evaluation only assesses changes in the clinically relevant severity levels 1 ("red") and 2 ("yellow"). Different alert categories will be analyzed separately, as well as in aggregated groups (1st degree), and aggregated over all alerts (2nd degree). See Fig. 2.

All analyses will take account of alert severity levels and the way the alert is handled by the GPs: 
- (i) Joint analyses of severity levels 1 and 2 and (ii) only severity level 1 to determine whether severity level 1 alerts were prioritized

- If the GP provides a note to confirm that the risk has been noted but that he or she has decided not to change the medication despite the alert, the analysis will be repeated (Justified alert, see Table 1).

The analysis will be performed for the overall patient population and include exploratory age-sex stratifications.

We will also analyze the percentage of enrolled patients for whom the bodily parameters kidney function, height and weight were entered. Finally, we will record the share of patients that had a printed medication plan and the share for whom a medication change had been documented.

Fidelity: We define "Implementation as intended" as the case when the user reacts to the triggers of all identified severity 1 alerts from the time of enrollment (T0) to two months later (T1), or provides notes explaining why he or she did not respond to an alert by changing the medication. A software change during the course of the study made it necessary for GPs to confirm that the patient's anamnesis had been completed before they saw the alerts. For this reason, we will conduct specific sensitivity analyses of interventions before and after that date, as well as of the GP population that confirmed completion of the anamnesis.

Tailoring: To assess whether eMMa was used in the physicians' spare time or during patient consultations, we will look at the days of the week that patients were enrolled. The ratio of enrollment rates on Fridays, weekends, and public holidays to the rest of the week will be calculated. The number of patients receiving the intervention per day will provide insight into whether GPs worked with eMMa en bloc or sporadically.

Data collection:

We will collect data from three different sources:

1. eMMa software saves changes made to a patient's medication by the GP and counts alerts at baseline and two months later.

2. KVWL delivers data on physicians. Physicians must be members of the KV to participate in the study, and work in the involved practices. The data will be delivered in aggregated form to ensure anonymity.

3. The BARMER statutory health insurer generates a data warehouse containing information on whether a patient was enrolled in eMMa by their GP or not. Aggregated information on the two patient groups will be provided in order that populations can be compared.

Table 2 provides an overview of the variables that contributed to outcomes. 


\section{Statistical Analysis:}

All outcomes will be presented using descriptive statistics. Mean, standard deviation, median, interquartile range and $95 \%$ confidence intervals will be provided for continuous, normally distributed variables. Frequency and percentages will be provided for binary and categorical variables. Poisson confidence intervals will be calculated for count variables that do not follow a normal distribution. For statistical testing, a significance level of alpha $=0.05(5 \%)$ and two-sided hypothesis testing (if not specified otherwise) will be applied. If variables are not normally distributed, non-parametric tests will be conducted. All analyses will be stratified according to the time of the practice's first enrolled patient according to the eMMa software version that was in use at that time. Impact of the COVID-19 pandemic will also be analyzed regarding regional shutdowns and incidences.

\section{Univariate Analysis}

Reach: To ensure data privacy, group comparisons in the Reach dimension will only be carried out on a descriptive level. To determine any association between enrollment rates and disrupting factors, we will conduct a time-series analysis and report the number of days on which such disruption occurred.

Dose: Differences in the fall in alerts between T0 (independent variable) and T1 (dependent variable) will be analyzed using the paired T-test and the Wilcoxon rank sum test for all types of alerts (Fig. 2, lowest level). The Chi ${ }^{2}$-Test will be used at $\mathrm{T} 1$ to compare documented vs non-documented and printed vs nonprinted items in eMMa.

Fidelity: The Chi²-Test will be used to test the decline in overall alerts to zero between T0 and T1 for the alert types displayed in Fig. 2 (medium level).

Tailoring: Differences in the number of patients on individual days of the week, between weekday and weekend as well as between quarters, months and years will be tested using the Chi ${ }^{2}$ test at T0.

Further multivariable (explanatory) analyses/subgroup analyses

Dose:

- Age- and sex-stratified analyses of the different alert categories (GP level) and stratification of the number of enrolled patients per GP

- Linear correlations between the different alert types will be represented using the Pearson correlation coefficient plus $\mathrm{p}$-values and corresponding scatterplots.

- Linear or logistic regression will be used to model the relationship between the reduction of all alerts (response variable) and both the different alert categories and patient characteristics (explanatory variables)

Fidelity: 
- Age- and sex-stratified analyses of medication regimen*dose*allergy alerts

Tailoring:

- Analyses of weekdays stratified according to number of enrolled patients per GP

Software: R and R Studio will be used to perform data quality checks, data transformation, statistical analysis, graphical visualization, and for reporting. The MySQL database will be used for data storage. To ensure data is imported and exported automatically, a link between the MySQL database and R will be created.

\section{Discussion}

This process evaluation will provide insight into the way GPs implement the AdAM intervention. By evaluating the kind of alerts that decrease and those that stay mostly unchanged, it can elucidate which patient groups are prioritized in terms of enrollment and how their medication is assessed. This will help explain findings in the main study. This is one of the first studies to evaluate GPs' decisions in the medication management process and - to the best of our knowledge - the first to support findings with log data from a CDSS.

However, there are some limitations to consider. The process evaluation relies on proper documentation by GPs since log data only show changes made in the system and may not display real life consequences. Still, these changes may be reflected in the potential improvement in primary and secondary outcomes without full visualization in the results of this process evaluation.

Since digital software like eMMa is continuously updated, this intervention will be affected by changes in the usability of the CDSS. Some of these may make it more difficult to compare the actions of GPs at different time points. For example, in the final version of the eMMa software, confirmation that the patient's anamnesis has been completed is required before alerts can be seen by GPs. If changes introduced during the study turn out to be relevant to the process evaluation, sensitivity analysis will be used to examine them. As log data can be used to compare time protocols for updates and technical difficulties that might have hampered the use of eMMa, we will be able to identify links between technical disruptions and the conduct of GPs.

\section{List Of Abbreviations}

AdAM: Anwendung für ein digital gestütztes Arzneimitteltherapie- und Versorgungsmanagement

CME: continuous medical education

cRCT: cluster-randomized controlled trial

eMMa: electronic medication management system 
GP: general practitioner

KVWL: Kassenärztliche Vereinigung Westfalen-Lippe

NSAID: non-steroidal anti-inflammatory drug

PIM: potentially inadequate medication

\section{Declarations}

\section{Ethics approval and consent to participate}

The AdAM study was approved by the ethics committee of the Association of Physicians in the German

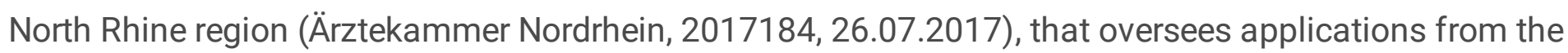
city of Wuppertal, where the lead manager of the AdAM study (BARMER statutory health insurance) is situated. Informed consent to participate is obtained by all patients or a caregiver on the patient's behalf.

\section{Consent for publication}

Not applicable.

\section{Availability of data and materials}

Data sharing is not applicable to this article as no datasets were generated or analyzed for this study protocol.

\section{Competing interests}

The authors declare that they have no competing interests.

\section{Funding}

This study was funded by the Innovation Fund of the German Federal Joint Committee (grant no 01NVF16006). The funder had no role in the design of the study, collection, analysis, or interpretation of data, or in the writing of the manuscript.

\section{Authors' contributions}

$\mathrm{RB}, \mathrm{DL}, \mathrm{KC}, \mathrm{MvdA}$ and CM designed the methodology of the process evaluation, with JKN, AP and SS contributing and adding new ideas. RB, DL, MvdA and CM wrote the original draft. All authors reviewed, edited, and agreed on the final version. DL, KC and NT are responsible for the statistical analysis. RB, DL and JKN provided the figures and tables. MvdA and CM supervised the work for this manuscript, and, together with JKN, WG and PKM, are responsible for project administration and funding acquisition.

\section{Acknowledgements}


We want to express special thanks to Phillip Elliott for the professional language review and to Annika Demuth and the RpDoc team for the description of the intervention and the functionality of eMMa.

Members of the AdAM study group: Lara Düvel, MA (BARMER, Wuppertal, Germany), Till Beckmann (BARMER, Wuppertal, Germany), Reinhard Hammerschmidt, Dipl.-Geogr., project manager (KVWL, Dortmund, Germany), Julia Jachmich, Dipl.-Pharm., Consulting Pharmacist (KVWL, Dortmund, Germany), Eva Leicher, Dipl.-Pharm., Consulting Pharmacist (KVWL, Dortmund, Germany), Benjamin Brandt, Project Administration (KVWL, Dortmund, Germany), Johanna Richard, PTA, prescription advice (KVWL, Dortmund, Germany), Frank Meyer, Dipl.-Pharm., MPH, Head of Department Corporate Development (KVWL, Dortmund, Germany), Dr. Mathias Flume, Dipl.-Pharm., MBA, Head of Division Member Service (KVWL, Dortmund, Germany), Thomas Müller, Dipl.-Ök. Member of Executive Board (KVWL, Dortmund, Germany), Prof. Dr. med. Ferdinand M. Gerlach, MPH (Institute of General Practice, Goethe-University, Frankfurt am Main, Germany), Dr. med. Beate S. Müller (Institute of General Practice, Goethe-University, Frankfurt am Main, Germany), Dr. med. Benno Flaig (Institute of General Practice, Goethe-University, Frankfurt am Main, Germany), Dr. Ana Isabel González-González, PhD (Institute of General Practice, Goethe-University, Frankfurt am Main, Germany), Truc Sophia Dinh (Institute of General Practice, GoetheUniversity, Frankfurt am Main, Germany), Peter Ihle (PMV research group, University of Cologne, Cologne, Germany), Ingo Meyer, MA (PMV research group, University of Cologne, Cologne, Germany), Prof. Dr. Hans Joachim Trampisch (Department of Medical Informatics, Biometry and Epidemiology, Ruhr University, Bochum, Germany), Renate Klaaßen-Mielke, Dipl.-Stat. (Department of Medical Informatics, Biometry and Epidemiology, Ruhr University, Bochum, Germany), Jale Basten, M.Sc. (Department of Medical Informatics, Biometry and Epidemiology, Ruhr University, Bochum, Germany), Bastian Surmann (Department of Health Economics and Health Care Management, Faculty of Health Science, Bielefeld University, Bielefeld, Germany), Prof. Dr. Holger Pfaff (Institute for Medical Sociology, Health Services Research and Rehabilitation Science, Department of Health Services Research, University of Cologne, Cologne, Germany), Vertr.-Prof. Dr. Ute Karbach (Technical University Dortmund, Department of Rehabilitation Sociology, Faculty of Rehabilitation Sciences, Dortmund, Germany) Karolina Beifuß, Dipl.Ök. (Center for Health Economics and Health Services Research, University of Wuppertal, Germany), Sarah Meyer, M.Sc. (Center for Health Economics and Health Services Research, University of Wuppertal, Germany), Prof. Dr. Daniel Grandt (Department of Internal Medicine. Clinic Saarbrücken, Germany), Simone Grandt (RpDoc Solutions GmbH, Saarbrücken, Germany).

\section{References}

1. United Nations. Department of Economic and Social Affairs, Population Division. World Population Ageing 2019: Highlights; 2019.

2. Masnoon N, Shakib S, Kalisch-Ellett L, Caughey GE. What is polypharmacy? A systematic review of definitions. BMC Geriatr. 2017;17:230. doi:10.1186/s12877-017-0621-2.

3. Guthrie B, Makubate B, Hernandez-Santiago V, Dreischulte T. The rising tide of polypharmacy and drug-drug interactions: population database analysis 1995-2010. BMC Med. 2015;13:74. 
doi:10.1186/s12916-015-0322-7.

4. Franchi C, Tettamanti M, Pasina L, Djignefa CD, Fortino I, Bortolotti A, et al. Changes in drug prescribing to Italian community-dwelling elderly people: the EPIFARM-Elderly Project 2000-2010. Eur J Clin Pharmacol. 2014;70:437-43. doi:10.1007/s00228-013-1621-6.

5. Duerden M, Avery T, Payne R. Polypharmacy and medicines optimisation: Making it safe and sound. London; 2013.

6. Cooper JA, Cadogan CA, Patterson SM, Kerse N, Bradley MC, Ryan C, Hughes CM. Interventions to improve the appropriate use of polypharmacy in older people: a Cochrane systematic review. BMJ Open. 2015;5:e009235. doi:10.1136/bmjopen-2015-009235.

7. Smith SM, Wallace E, O'Dowd T, Fortin M. Interventions for improving outcomes in patients with multimorbidity in primary care and community settings. Cochrane Database Syst Rev. 2016;3:CD006560. doi:10.1002/14651858.CD006560.pub3.

8. Miller WL, Crabtree BF, Nutting PA, Stange KC, Jaén CR. Primary care practice development: a relationship-centered approach. Ann Fam Med. 2010;8(Suppl 1):68-79. doi:10.1370/afm.1089. S92.

9. Wierenga D, Engbers LH, van Empelen P, Duijts S, Hildebrandt VH, van Mechelen W. What is actually measured in process evaluations for worksite health promotion programs: a systematic review. BMC Public Health. 2013;13:1190. doi:10.1186/1471-2458-13-1190.

10. Moore GF, Audrey S, Barker M, Bond L, Bonell C, Hardeman W, et al. Process evaluation of complex interventions: Medical Research Council guidance. BMJ. 2015;350:h1258. doi:10.1136/bmj.h1258.

11. World Health Organization. The use of the WHO-UMC system for standardised case causality assessment.

12. Guyatt GH, Alonso-Coello P, Schünemann HJ, Djulbegovic B, Nothacker M, Lange S, et al. Guideline panels should seldom make good practice statements: guidance from the GRADE Working Group. J Clin Epidemiol. 2016;80:3-7. doi:10.1016/j.jclinepi.2016.07.006.

13. Dreischulte T, Donnan P, Grant A, Hapca A, McCowan C, Guthrie B. Safer Prescribing-A Trial of Education, Informatics, and Financial Incentives. N Engl J Med. 2016;374:1053-64. doi:10.1056/NEJMsa1508955.

14. Muth C, Harder S, Uhlmann L, Rochon J, Fullerton B, Güthlin C, et al. Pilot study to test the feasibility of a trial design and complex intervention on PRloritising MUltimedication in Multimorbidity in general practices (PRIMUMpilot). BMJ Open. 2016;6:e011613. doi:10.1136/bmjopen-2016-011613.

15. O'Mahony D, O'Sullivan D, Byrne S, O'Connor MN, Ryan C, Gallagher P. STOPP/START criteria for potentially inappropriate prescribing in older people: version 2. Age Ageing. 2015;44:213-8. doi:10.1093/ageing/afu145.

16. van den Bussche $H$, Koller D, Kolonko T, Hansen H, Wegscheider K, Glaeske G, et al. Which chronic diseases and disease combinations are specific to multimorbidity in the elderly? Results of a claims data based cross-sectional study in Germany. BMC Public Health. 2011;11:101. doi:10.1186/14712458-11-101. 
17. Quinzler R, Freitag MH, Wiese B, Beyer M, Brenner H, Dahlhaus A, et al. A novel superior medicationbased chronic disease score predicted all-cause mortality in independent geriatric cohorts. J Clin Epidemiol. 2019;105:112-24. doi:10.1016/j.jclinepi.2018.09.004.

18. Charlson M, Szatrowski TP, Peterson J, Gold J. Validation of a combined comorbidity index. J Clin Epidemiol. 1994;47:1245-51. doi:10.1016/0895-4356(94)90129-5.

19. Mukherjee B, Ou H-T, Wang F, Erickson SR. A new comorbidity index: the health-related quality of life comorbidity index. J Clin Epidemiol. 2011;64:309-19. doi:10.1016/j.jclinepi.2010.01.025.

\section{Tables}

Due to technical limitations, the tables are only available as a download in the supplemental files section.

\section{Figures}

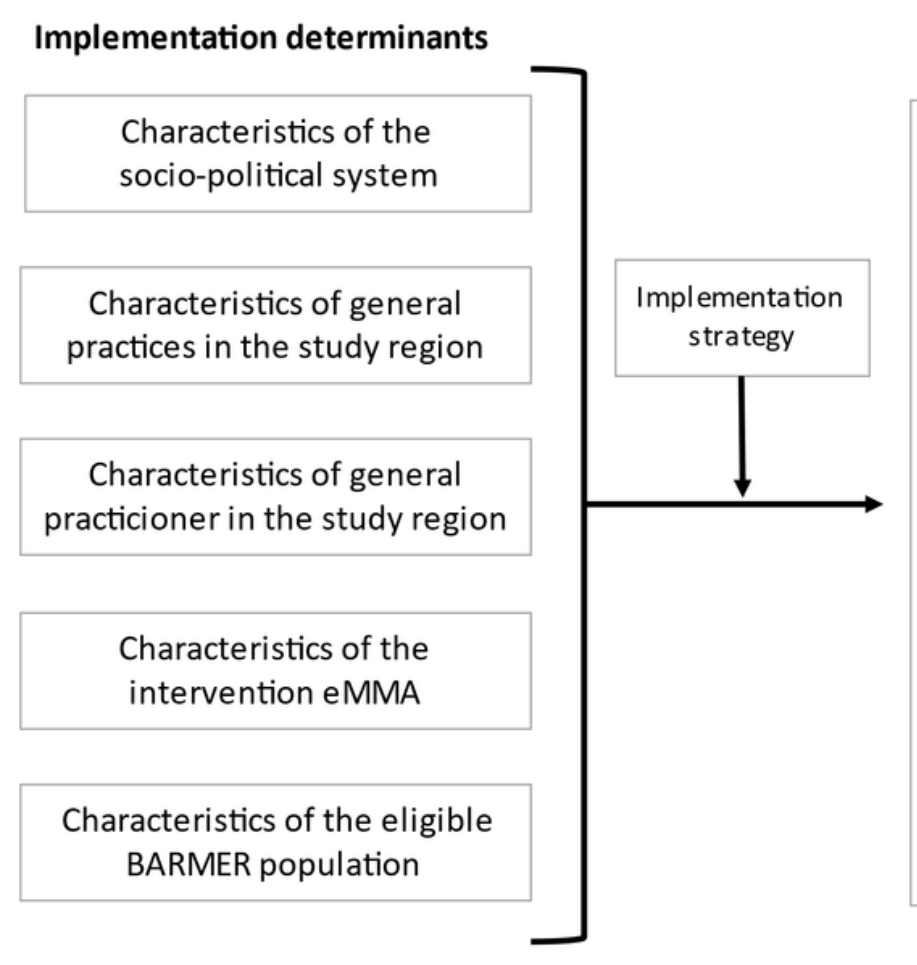

Implementation process

\begin{tabular}{|c|}
\hline Adoption: \\
Strategy of inviting general \\
practices \\
\hline \begin{tabular}{c} 
Implementation: \\
Reach on practice, GP and patient \\
level; \\
Dose: How comprehensive is the \\
intervention applied? \\
Fidelity: Was the intervention \\
implemented in a way that makes \\
success possible? \\
Tailoring: How did physicians \\
implement the intervention to better \\
fit to their daily routine? \\
\hline \\
Continuation: \\
Maintenance \\
\hline
\end{tabular} \\
\hline
\end{tabular}

\section{Figure 1}

Theoretical framework of the process evaluation based on Wierenga et al 2013.[9] Maintenance of the results is part of another publication of the AdAM study. 


\section{Total alerts}

Medication-

regimen-

related
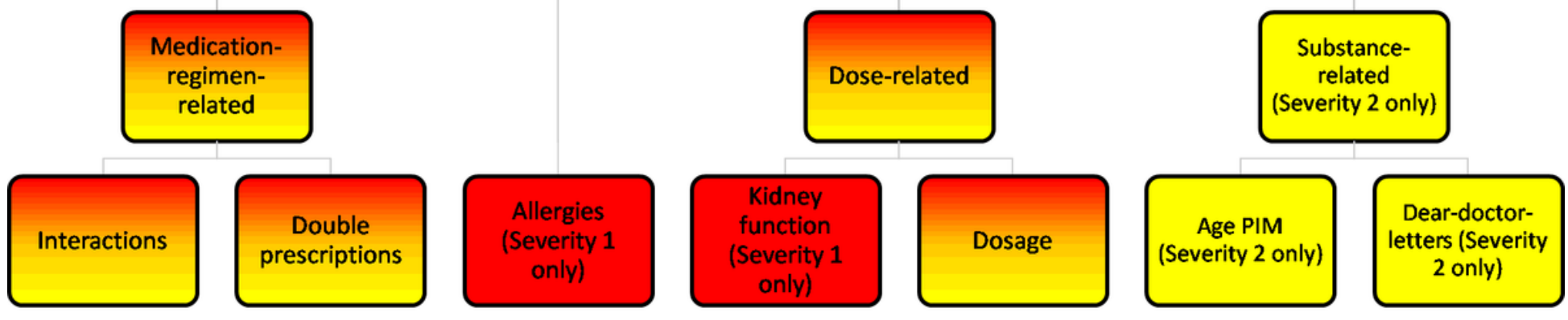

Figure 2

Flowchart of analyzed alerts. Red background indicates severity 1 in that category; yellow background indicates severity 2 in that category; red and yellow background indicates that both alerts may be applicable in the category.

\section{Supplementary Files}

This is a list of supplementary files associated with this preprint. Click to download.

- Tables.pdf

- TIDieRchecklist.docx 